\title{
COMPOUNDING AND PROCESSING HYDRO-BIODEGRADABLE PLASTIC FILMS FOR PLASTIC WASTE REDUCTION. PART II: THERMAL AND CHEMICAL PRINTING OF VIRGIN/WASTE POLYMERIC BLENDS
}

\author{
MASUMAH AL-QASSIMI, HAJAR JAWAD KARAM, \\ MAJED HAMEED AL-WADI \& SULTAN MAJED AL-SALEM \\ Environment \& Life Sciences Research Centre (ELSRC), Kuwait Institute for Scientific Research (KISR), Kuwait
}

\begin{abstract}
In an effort to develop a biodegradable blend that could withstand arid environmental conditions; and standardise the use of biodegradable plastic films originating from hydro-biodegradable blends, this project was initiated by the Kuwait Institute for scientific Research. In this work, linear low-density polyethylene (LLDPE) was compounded with a $3 \mathrm{wt} \%$ thermoplastic starch (TPS) resin and by the same amount with a pro-degradant chemical, using three different techniques that are standardised in the industry of plastics conversion. These are extrusion by single screw compounding, twin screw compounding and blown-filming machine as well. The materials were studied in comparison to the virgin LLDPE, as well as, formulated blends with plastic solid waste (PSW) materials, also in an effort to determine the impact such chemicals and pro-degradants have on the municipal solid waste (MSW) stream. Firstly, mechanical properties were studied using a universal testing machine by applying ASTM 882-12 and compared to other market grades available within localities. Furthermore, the thermogravimetric behaviour and infrared spectroscopy (IR) were established and have resulted in a clear shift within the onset and end set temperatures, in comparison to other commercial grades. This study establishes a baseline for compounds that could be used in the future as a biodegradable plastic film in an effort to combat plastic solid waste (PSW) accumulation in developing countries.

Keywords: biodegradation, polyester, starch, polymer waste, waste management.
\end{abstract}

\section{INTRODUCTION}

Packaging is a process that encloses or covers goods intended for shipment, storage, sale and use. Polyethylene (PE) is the commonly used polymer in packaging. It is converted into packaging materials by thermal processes such as film blowing, sheet extrusion, or extrusion coating [1]. The packaging sector is the largest consuming sector by demand for all polymer types and resin. Fig. 1 shows the global demand for plastics by sector where packaging, building and textiles represents the major share of the market by $34 \%, 17 \%$ and $10 \%$, respectively [2]. The increase in dependency on plastics has led to an increase in the amount of plastic solid waste (PSW) generation, which is estimated to be 260 million tonnes per annum on a global basis [3].

The properties of products play an important role in improving the quality and shelf-life of the food in terms of food packaging and other products where plastic covers are typically used [4]. A key requirement for food packaging materials is that they should not emit substances that interfere with the product, i.e. cause odour and taste. EQUATE Petrochemical Company in the State of Kuwait currently produces approximately $825 \mathrm{ktpa}$ of linear low-density polyethylene (LLDPE) and high-density polyethylene (HDPE) with an expected increase in market demand in the coming years. Plastic converters that make shopping bags, trash bags, agricultural mulches, greenhouse covers and many others use a 
significant portion of these polymers locally most of which end up as waste. The generation of PSW in Kuwait is estimated at around 150 to $200 \mathrm{ktpa}$ and is predicted to grow as a result of population growth and high living standards in Kuwait. The predominant technique of disposal of solid waste (SW) in Kuwait is landfill burial and with PSW taking very long time to effectively degrade in the environment. PSW accumulation and management is perceived to be one of the main issues facing both government and private sector parties. Recent estimates have shown that Kuwait produces $825 \mathrm{ktpa}$ of PE and imports more than $25 \mathrm{ktpa}$ of virgin resin most of which are used in the packaging industry and the production of bags [5].

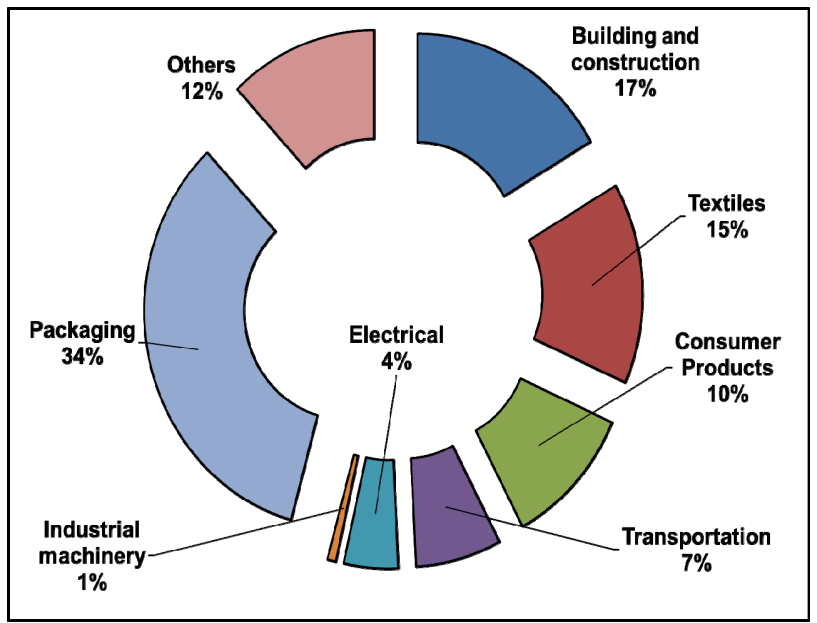

Figure 1: Plastic resin global demand by sector. (Source: Redrawn from Parker, 2018 [2].)

In this work, linear low-density polyethylene (LLDPE) was compounded with a $3 \mathrm{wt} . \%$ thermoplastic starch (TPS) resin and by the same amount with a pro-degradant chemical, using three different techniques that are standardised in the industry of plastics conversion. These are extrusion by single screw compounding, twin screw compounding and blown-filming machine as well. The materials were studied in comparison to the virgin LLDPE, as well as, formulated blends with plastic solid waste (PSW) materials, also in an effort to determine the impact such chemicals and pro-degradants have on the municipal solid waste (MSW) stream.

\section{EXPERIMENTAL}

LLDPE (grade EFDC-7050) was used as received in a translucent pellet form with a reported density of $0.918 \mathrm{~g} \mathrm{~cm}^{-3}$ [1]. The LLDPE polymer was compounded with a $3 \mathrm{wt} . \%$ thermoplastic starch ECOVIO (TPS) resin and by the same amount with a PDQ-M Willow Ridge Plastics (Inc.) pro-degradant chemical, using three different techniques that are standardised in the industry of plastics conversion. These techniques are namely, (a) single screw extrusion, (b) twin screw extrusion, and (c) blow-filming which are the most common among commercial film production lines, and have been chosen to study the effect of processing and melting on the materials. Throughout the rest of this paper, these techniques will be coded as follows: a) SSE, (b) TSE, and (c) BF, respectively. The PDQ-M pro-degradant chemical was used as received in the form of tan pellets and a 
melting point $\left(\mathrm{T}_{\mathrm{m}}\right)$ equal to $110^{\circ} \mathrm{C}$. The thermoplastic starch (TPS) was used as white translucent pellets and was certified by the manufacturer in accordance with EN17033 with a $\mathrm{T}_{\mathrm{m}}$ equal to $120^{\circ} \mathrm{C}$. SSE was performed using a Dolci extruder (L/D ratio $1 / 28$ ) using $10 \mathrm{~kg}$ as master batch mix. The blends with TPS were conducted in the same mix ration as the oxo-additives, to have a comparable baseline between the materials. Mixing with palletized PSW was also conducted in the same blend ration of 3 wt.\%. Furthermore, BF was conducted with the same formulations aforementioned using a Windsor (monolayer) blown-film coupled with an L/D (1/28) single screw extruder operated under 6 bars of pressure with an RPM of maintained between 75 and 80. TSE was performed using a Kung Hsing (1:28, L/D ratio, 6 bars) operated in co-rotation with a maximum speed of 75 RPM maintained throughout the experimental work. The TPS samples were extruded in a standalone Brabender SSE which was used in the compounding process of the samples. All samples were extruded as sheets of $100 \mu \mathrm{m}$ thickness meeting standard market specifications and as an average of major film applications. The samples appeared consistent with no visual fractures or crazing and were comparable to each other by touch. The mechanical properties were studied following test protocol of ASTM 882 using a Tinius Olsen UTM machine as depicted previously in Al-Salem et al. [1]. Variation in processing and cutting was negligible over the length of some samples between the width and thickness, accounting for less than $1 \%$ of the total number of samples measured. The variation for the samples was not greater than $\pm 0.05 \mathrm{~mm}$ for width and $\pm 0.02 \mathrm{~mm}$ for thickness, and a constant width of $12.50 \mathrm{~mm}$ and $0.10 \mathrm{~mm}$ for thickness was taken. Thermogravimetric analysis (TGA) was conducted for all compounded compounded LLDPE and PSW films without and with TPS and PDQ-M under nitrogen atmosphere in a Mettler-Toledo TGA Unit coupled with a StarE software using $7.5 \mathrm{mg}$ sample mass and at a heating rate of $15^{\circ} \mathrm{C} \mathrm{min}{ }^{-1}$ from room temperature to $600^{\circ} \mathrm{C}$. Following previously conducted work [6], [7], the samples were thermally monitored via infrared spectroscopy using Fourier transform infrared spectroscopy (FTIR) in attenuated total reflectance (ATR) mode. The film samples were mounted on the crystal of the machine's holder, and the spectra after each sample unloading was taken. Background spectra were collected before each measurement. An initial frequency range of 500 to $5000 \mathrm{~cm}^{-1}$ with an average $32 \mathrm{scan}$ of a resolution of $4 \mathrm{~cm}^{-1}$ was used at room temperature. The ketone carbonyl bond at 1714 $\mathrm{cm}^{-1}$ was considered and its bond index $(\mathrm{KCBI})$ was estimated as the ratio of the carbonyl group, band and that of the PE band thus

$$
K C B I=\frac{I_{1714}}{I_{1470}} .
$$

The $1470 \mathrm{~cm}^{-1}$ absorbance is taken as the PE band. Upon studying the evolution of the carbonyl band, the other component indices were calculated and the main products according to literature [8] are namely: Lactone (L), Ester (E)/Aldehydes (A), Carboxylic Acid (CA), and Vinylidene (V). An index was allocated to each of these products, which was determined to measure the degree of thermal degradation in relation to the PE band reference point, bearing in mind that reference bands could vary with the type of polymer film and the type of test conducted. The target bands may differ, hence at first the peaks must be tracked and calculated using the FTIR results of the control sample

$$
L B I, E B I / A B I, C A B I, V B I=\frac{I_{n}}{I_{1470}},
$$


where $n$ stands for I1714, I1770, I1733, I1698, and I888 which are which are associated with band absorbance at $1714,1770,1733,1698,888$, respectively following previously depicted methodology shown elsewhere [9].

\section{RESULTS AND DISCUSSION}

The extrusion profile with respect to temperatures of various temperature zones against for all three compounding techniques showed a typical processing conditions trend that was reported previously by other authors [1], [9]. The relationship between the polymer structure and its mechanical behaviour should not be disregarded, and several studies have been carried out over the last few years to investigate the impact of molecular structures on the overall characteristics of PE, in particular its mechanical properties [10]-[12]. LLDPE is typically produced by co-polymerisation of ethylene and such higher alpha olefins, including butane, hexane or octane, at lower temperatures and pressures. The copolymerisation process gives LLDPE a narrower molecular weight distribution, linear structure and significantly different rheological properties than traditional LDPE polymer. The sample preparation technique was taken into account when comparing the LLDPE data from this study with data from other PE studies. One of the earliest studies conducted on LLDPE was that of Dumoulin et al. [13] who examined the tensile properties of two series of LLDPE with isotactic PP. The blends were prepared using TSE at $245^{\circ} \mathrm{C}$. The composition dependence of Young's modulus and of the strain at break versus concentration of PP in LLDPE blends was reported. The strain at break of LLDPE-1/PP $(100 / 0)$ and LLDPE-2/PP (100/0) blends were 6.5\% and 8.5\%, respectively. The Young's modulus measured for LLDPE-1/PP (100/0) and LLDPE-2/PP (100/0) were approximately 0.30 and $0.13 \mathrm{GPa}$, respectively. Change in mechanical properties of LLDPE polymer and molecular structure due to degradation were followed in depth by Naddeo et al. [14]. Mechanical parameters were also correlated to exposure time and carbonyl index. The LLDPE pellets were moulded in a hot press at $240^{\circ} \mathrm{C}$ to form films of $130 \mu \mathrm{m}$ thickness. The stress-strain curve of the initial LLDPE sample before being exposed to accelerating weathering derived the mechanical properties of interest namely: Elastic modulus (120 $\mathrm{MPa})$, stress at yield point (8.3 MPa), stress at break (9 MPa), strain at break (1690\%) and toughness $(323 \mathrm{MPa})$. The mechanical properties in terms of Young's modulus, yield stress, impact strength, and elongation at break of pure and zeolite filled LLDPE blends were studied by Biswas et al. [15] using a UTM of Instron 4465 , at $25^{\circ} \mathrm{C}$ and $30 \%$ humidity. The pure LLDPE and its blends were prepared using a Brabender twin-screw extruder (PL 2000) with L/D screw dimension of 16 at temperature gradient of $170^{\circ} \mathrm{C}$ in the feeding zone, $180^{\circ} \mathrm{C}$ in the compression zone, $190^{\circ} \mathrm{C}$ in the metering zone and $200^{\circ} \mathrm{C}$ at die. The screw's rotation speed was kept between 60 and $70 \mathrm{rpm}$. Two specimen types, dumbbell and film, were prepared for the mechanical properties' measurement at different crosshead speeds. For the film specimens (dimensions: $15 \times 0.4 \times 165 \mathrm{~mm}^{3}$ ) in particular, the initial grip distance was between 50 and $500 \mathrm{~mm} / \mathrm{min}$. The stress-strain curves of the pure LLDPE film showed that elongation at break for $5 \mathrm{~mm} / \mathrm{min}$ exceeded $1000 \%$ (i.e. sample can be elongated up to $500 \mathrm{~mm}$ ) and a similar behaviour was observed at $500 \mathrm{~mm} / \mathrm{min}$. In addition, for the pure LLDPE, the Young's modulus obtained for 50 and $500 \mathrm{~mm} / \mathrm{min}$ strain rates was 120 and $127 \mathrm{MPa}$, respectively. The yield stress measured for the pure LLDPE at strain rates of 50 and $500 \mathrm{~mm} / \mathrm{min}$ were 6.8 and $9.5 \mathrm{MPa}$, respectively. The elongation at break for the pure LLDPE was $200 \%$ and $1000 \%$ (i.e. machine can elongate the sample up to $500 \mathrm{~mm}$ ) at strain rates of 50 and $500 \mathrm{~mm} / \mathrm{min}$, respectively.

The comprehensive literature review conducted on the mechanical properties of LLDPE, LDPE, and HDPE and their techniques of compounding provided a solid basis for 
comparison of the films examined in this study. The films compounded were all comparable to market standards which renders them applicable for use as a carrier film and packaging product [1]. The polymer blends compounded using the SSE, TSE and BF showed slight variations in the Young's modulus (Fig. 2). The compounding method and the incorporation of TPS and PDQ-M additives into the blends altered the mechanical properties of the samples in comparison to the pure LLDPE and PSW. The highest calculated Young's modulus value for the pure LLDPE was $5048 \mathrm{MPa}$ compounded using Blow-filming/extrusion which was much higher than the other LLDPE blends with and without additives (Fig. 2). The highest calculated Young's modulus value for PSW (100) compounded using twin screw extrusion was $4547 \mathrm{MPa}$ which was slightly higher than that reported for its blends with TPS and PDQ. This showed that their relative compounding techniques allowed the pure blends (100 wt.\%) to exhibit higher stiffness and less rapid deformation. In general, the Young's modulus reported in the study are several folds higher than those reported in literature. This could be attributed to the high content of plasticisers in LLDPE and PSW which gave increased flexibility to the compounded films.

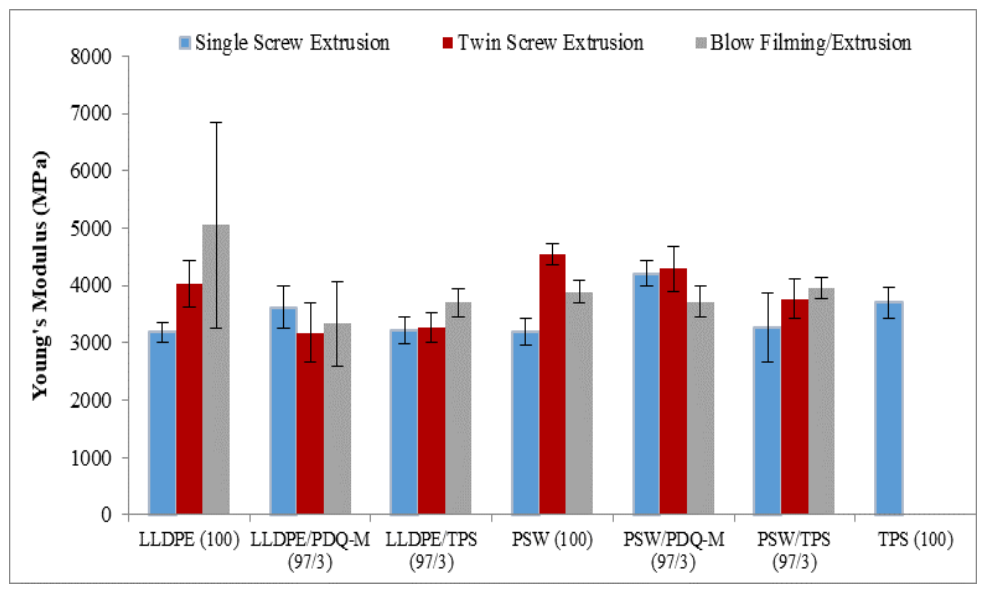

Figure 2: Young's modulus (MPa) measured for the compounded LLDPE and PSW films without and with TPS and PDQ-M.

The estimated Young's modulus value of the TPS compounded in single screw extrusion was $3696 \mathrm{MPa}$. The thermal and mechanical properties of a comparative TPS commercial grade blend with PP were investigated by Pang et al. [16]. Sheet extrusion was performed in a Berlyn model single-screw extruder with an L/D ratio of 31:1 and processing temperature range of $150-170^{\circ} \mathrm{C}$, and screw speed of $50 \mathrm{rpm}$. Tensile properties of dumbbell specimens were determined with an Instron model 3366 tester according to ASTM D 638, at a cross-head speed of $50 \mathrm{~mm} / \mathrm{min}$. The tensile properties of the extruded pure TPS (100) sheets showed the lowest tensile Strength (5-7 MPa), Elongation at break $(6 \%-16 \%)$ and relatively high Young's modulus (540-660 MPa) values compared to the other blends. This is due to the high starch content in the formulation (biobased content above 50\%) compared to NTS/PP, AWS/PP, and AWT/PP blends (biobased content $35 \%-37 \%$ ). This finding is consistent with previous research, where the introduction of starch granules to synthetic polymers influenced the mechanical properties by reducing tensile strength and elongation at break, while the Young's modulus increased as the starch 
content increased due to the stiffing effect of the granules [17]. In addition, according to Azhari and Wong [18] a high content of starch in the sample can cause poor dispersion as starch granules agglomerate, leading to a reduction in mechanical properties. Polystyrene/TPS blends were prepared by melt mixing and characterised by TGA, FTIR and tensile tests (strength, modulus, elongation at break and tenacity) [19]. PS/TPS blends were prepared using an intensive Brabender type mixer with two counter-rotating roller rotors at a mixing temperature of $155^{\circ} \mathrm{C}$, speed rotation of $150 \mathrm{RPM}$ and mixing time of 10 min. Films were prepared by compression moulding with a thickness of 300-500 mm. For TPS $(0 / 100)$ blend, the stress-strain curve, conditioned at 30\% Relative Humidity (RH), showed a linear elastic behaviour and a subsequent plastic deformation but without strain hardening. The curves obtained from the tensile tests were used to measure the mechanical properties of TPS at $30 \% \mathrm{RH}$ and $25^{\circ} \mathrm{C}$. Tensile Strength of $9.5 \mathrm{MPa}$, Elongation at break of $3.4 \%$ and Young's modulus of $790 \mathrm{MPa}$ were measured at $30 \% \mathrm{HR}$. The effect of relative humidity on the mechanical behaviour was studied further at $60 \%$ and $75 \% \mathrm{RH}$ to test the environment impact on the mechanical behaviour of TPS. Humidity has been found to have a major impact on TPS' mechanical properties as it serves as a plasticiser for TPS, lowering the modulus and tensile strength of its blends and increasing the elongation at breakage. The impact of TPS on lowering Young's modulus was mainly evident in all the LLDPE blends as see in Fig. 2.

The degradation of pure LDPE and its blend with a starch-based pro-degradant additive (PSH) (25 wt.\%) under various degrading media was previously studied [20]. The LDPE/PSH blend was compounded in a Brabender Plasticorder (Mixer 50E) for $10 \mathrm{~min}$ at temperature of $1408^{\circ} \mathrm{C}$ and mixing speed of $60 \mathrm{rpm}$. The stress-strain plots for the LDPE/PSH before aging showed that the incorporation of the pro-degradant additive caused a drop in mechanical properties. The reported tensile strength of the pure LDPE and LDPE/PSH blends containing $25 \mathrm{wt} \%$ of PSH before aging were $15.30 \pm 0.09 \mathrm{MPa}$ and $11.40 \pm 0.10 \mathrm{MPa}$, respectively. The pure LDPE and LDPE/PSH blend recorded elongation at break of $338 \pm 2.12 \%$ and $118 \pm 6.8 \%$, respectively, before aging. The application of weathering tests to determine the degradation profile polymers used in plastic production has been studied to a great extent in literature [21]-[24]. The mechanical and physical properties of PSW in the form of discarded plastic films were tested along the pure LLDPE polymer [24]. Dry blends of LLDPE and PSW were extruded, and blown using SSE $(\mathrm{L} / \mathrm{D}=30)$. Pure LLDPE/PSW ratio of the compounded blends were $100 / 0,95 / 5,90 / 10$, $75 / 25,50 / 50,25 / 75,10 / 90$ and 0/100. The mechanical behaviour of pure LLDPE (100) and PSW (100) blends in terms of the Young's modulus, stress and elongation at break was assessed and compared to the mechanical assessment conducted in this study. The Young's modulus measured for the pure LLDPE and the PSW blends was 21 and $72 \mathrm{MPa}$, respectively. The PSW blends also showed a decreasing trend in the modulus as the waste content increased signifying that the plastic film waste acts as a degrading agent to the pure LLDPE. The pure LLDPE and PSW samples had a relatively similar stress at break values of approximately $19 \mathrm{MPa}$ and $18 \mathrm{MPa}$, respectively. The pure LLDPE an PSW samples had a strain at break values of $1478 \%$ and $500 \%$, respectively. From the study a clear decrease in mechanical properties was observed as a result of both the waste material and natural weather exposure. Al-Salem et al. [23], [24] also evaluated mechanical and physical (optical) properties of pure LLDPE and its blends with PSW under accelerated weathering. They further investigated in depth the effect of die head temperatures (DHTs) at compounding stage on the degradation of the compounded blends [24]. Samples properties were tested at three DHTs in the extruder section: $175^{\circ} \mathrm{C}, 185^{\circ} \mathrm{C}$ and $195^{\circ} \mathrm{C}$ and those reported at $195^{\circ} \mathrm{C}$ in particular were used in comparison to the current study for samples 
compounded using BF/ESS DHT of $190-200^{\circ} \mathrm{C}$. The Young's modulus and stress at break values reported for pure LLDPE were similar at all DHTs reaching values of a round $62 \mathrm{MPa}$ and $18 \mathrm{MPa}$, respectively, at $\mathrm{DHT}=195^{\circ} \mathrm{C}$. The strain at break reported at $195^{\circ} \mathrm{C}$ DHTs was $1500 \%$. On the other hand, the Die Head Blown-film method used by the extruder altered mechanical properties of the samples when waste fractions were added to the blends in comparison to the pure LLDPE. The Young's modulus and stress at break values reported for $\mathrm{LLDPE} / \mathrm{PSW}(10 / 90)$ at $\mathrm{DHT}=195^{\circ} \mathrm{C}$ were $20 \mathrm{MPa}$ and $14 \mathrm{MPa}$, respectively. A strain at break value of $400 \%$ was reported at $195^{\circ} \mathrm{C}$ DHTs for the LLDPE/PSW (10/90) blend.

For the SSE, TSE and BF techniques (used in this work) the ranges of Young's Modulus measured for the pure LLDPE, LLDPE/PDQ-M (97/3), and LLDPE/TPS (97/3) were 3180-3620, 3176-4027, and 3333-5048 MPa, respectively. For the PSW, PSW/PDQ-M (97/3), and PSW/TPS (97/3) blends compounded using SSE, TSE, and BF techniques the ranges of Young's Modulus measured were 3185-4210, 3766-4547, and 3718-3951 MPa, respectively. The tested films and PSW in particular were seen to have relatively high modulus values when compared to LLDPE films in literature and have surpassed the stiffness measurements required for the local market. The tested PSW films have shown potential for utilising the plastic waste in the local plastic production industry. The modulus is found to dependant on the individual modulus values of the materials used in the blend and is showing an increasing trend when certain polyolefin or polyester polymers are present in the blend in proportion to their amount [25]. In the study, Young's modulus was found not to be related to waste content in the blend especially when chemical additives were added due to the low TPS and PDQ-M percentage (3 wt.\%).

All samples experienced a major weight loss stage in the TGA associated with the degradation of the synthetic polymer such as LLDPE. In the case of LLDPE and PSW blends with TPS two major weight loss stages were expected the first being that of the synthetic polymer and the second is typically due to the degradation of the starch. Also in this study, ATR-FTIR was used as a qualitative assessment method to aid in polymer identification based on their specific absorption bands. Each FTIR spectrum of LLDPE and PSW virgin and compounded films were compared with reference spectra from the spectral database of the FTIR FDM Library. The program shows a variety of compounds correlated with correlation coefficients, of which the FTIR spectra matches the spectra of the analysed film sample. In order to determine the samples degree of degradation, the region of carbonyl group's absorption band (1680-1820 $\left.\mathrm{cm}^{-1}\right)$ was investigated for all pre-degradable tested films. Martínez-Romo et al. [26] tested the changes in structural properties of HDPE, LDPE, bio-degradable polyethylene (PE-BIO), and oxo-degradable PE films without exposure to UVB radiation. The infrared spectra of the tested films showed the PE characteristics bands: $2772-3038 \mathrm{~cm}^{-1}$ (the stretching vibration of Carbon-Hydrogen $(\mathrm{CH})$ ), $1440-1490 \mathrm{~cm}^{-1}$ (shaking and rocking vibration of methylene $\left(\mathrm{CH}_{2}\right), 700-750 \mathrm{~cm}^{-1}$, respectively [27]. The PE-BIO in particular showed peaks at $700-750 \mathrm{~cm}^{-1}$ and $1440-1490$ $\mathrm{cm}^{-1}$ due to the polymers' linear structure, while for LDPE and PE-OXO, these same bands were divided because they represent branched polymers. The IR spectra of the PE-BIO films showed absorption band at $1740 \mathrm{~cm}^{-1}$ (stretching vibration of the carbonyl group $(\mathrm{C}=\mathrm{O})$ and $\mathrm{PE}-\mathrm{OXO}$ film showed absorption band at $909 \mathrm{~cm}^{-1}$ (stretching vibration of the vinyl group $\left(\mathrm{CH}_{2}=\mathrm{CH}\right)$ ). Such functional groups are found in chromophores and are considered precursors of PE photochemical reactions [28]. The carbonyl and vinyl groups are considered the PE's key photo-oxidation products [29]; thus, the carbonyl index (ICO) and the vinyl index (IV) are parameters used to determine the effect of UV-B radiation in PE films [30]. The PE-BIO film IR spectrum without exposure to UV-B radiation presented 
the characteristic carbonyl group IR band $\left(1640 \mathrm{~cm}^{-1}\right)$ which indicated that the PE-BIO content is oxidised during the manufacturing process.

FTIR spectra (see Fig. 3 for an example) showed that all LLDPE and PSW films compounded without TPS and PDQ-M using the three compounded techniques presented major characteristic PE absorbance bands that are located at $2916 \mathrm{~cm}^{-1}$ and $2846 \mathrm{~cm}^{-1}(\mathrm{C}-\mathrm{H}$ stretching asymmetric and symmetric vibrations), $1462 \mathrm{~cm}-1$ (crystalline phase of $\mathrm{CH}_{2}$ bend), and $719 \mathrm{~cm}^{-1}\left(\mathrm{CH}_{2}\right.$ rocking vibrations). A weak absorption band at $1471 \mathrm{~cm}^{-1}\left(\mathrm{CH}_{2}\right.$ scissoring vibrations) was also observed for all pre-degradable films suggesting that degradation is not occurring. The $1470 \mathrm{~cm}^{-1}$ and $798 \mathrm{~cm}^{-1}$ peaks are used to identify and quantify the presence of PE [31]. In general, the detected bands follow a polyethylene (PE) spectrum as previously depicted by past works [32]. In addition, the detected bands are in agreement with the FTIR spectra of unexposed [6], commercial LDPE grade material [33] and IR spectra of the control LLDPE film blends [34] to predict the lifetime of oxo-degradable polyolefin and biodegradable polymer films. FTIR spectra obtained for LLDPE are comparable to published spectra for respective polymer blend [33]-[35].

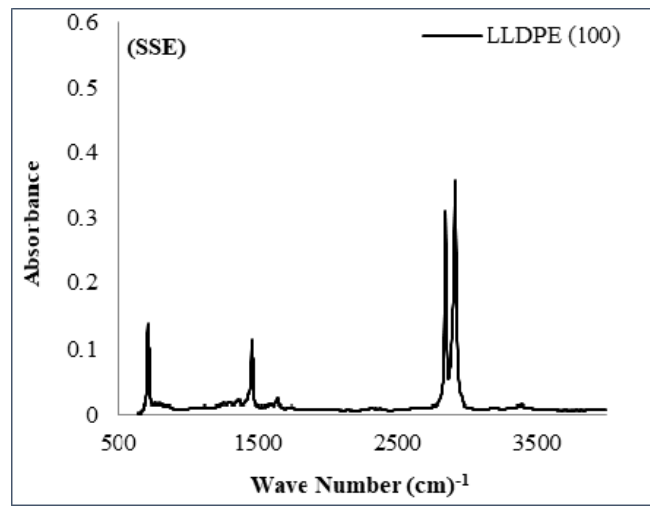

(a)

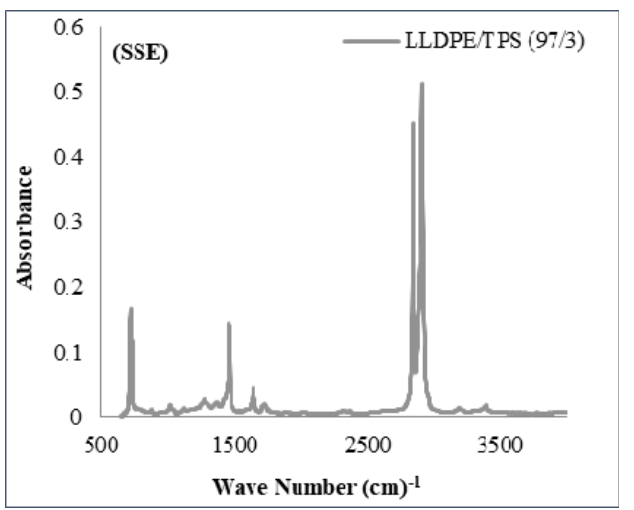

(b)

Figure 3: SSE samples showing the spectra obtained with ATR analysis for (a) LLDPE; and (b) LLDPE/TPS).

In general, compared to LLDPE and PSW the peak locations and peak shapes of the blends were alike, however, absorbance of LLDPE/TPS was higher than LLDPE and LLDPE/PDQ-M for single screw extrusion. In addition, it was also observed that absorbance of LLDPE was lower than that of its blends compounded with PDQ-M and TPS using blow-filming/extrusion. On the other hand, peak locations, peak shapes and absorbance of all films compounded in twin screw extrusion were alike for all tested films. The LLDPE films with PDQ-M also showed absorption band referent to PE $2916 \mathrm{~cm}^{-1}$ $\left(\mathrm{CH}_{2}\right.$ asymmetric stretch bonds), $1462 \mathrm{~cm}^{-1}$ (crystalline phase of $\mathrm{CH}_{2}$ bend), and $719 \mathrm{~cm}^{-1}$ (crystalline phase of $\mathrm{CH}_{2}$ rock). In general, very weak absorption band closer to $1715 \mathrm{~cm}^{-1}$ (stretching vibration of $\mathrm{C}-\mathrm{O}$ groups) that indicates the presence of carbonyl $(\mathrm{C}=\mathrm{O})$ reflecting the esters of biopolymers and the possible oxidation of the LLDPE and PSW compounded films. In literature, a typical FTIR spectra of PE+AD (AD: oxo-degradable additive) blend will have main peaks corresponding to hydroperoxides $\left(3200 \mathrm{~cm}^{-1}\right)$, alcohols $\left(3416 \mathrm{~cm}^{-1}\right)$, Ketones $\left(1715 \mathrm{~cm}^{-1}\right)$, aldehyde $\left(1725 \mathrm{~cm}^{-1}\right)$, carboxylic acids $(1710$ and 1715), noncyclic/cyclic esters $\left(1735\right.$ and $\left.1785 \mathrm{~cm}^{-1}\right)$ and double bonds $\left(909 \mathrm{~cm}^{-1}\right)$ [36]. 
The spectra of LLDPE/PDQ-M (97/3) and PSW/PDQ-M (97/3) had appreciable differences due to the low PDQ-M\% in these blends. For TPS, weak absorption band at $2918 \mathrm{~cm}^{-1}$ $\left(\mathrm{CH}_{2}\right.$ asymmetric stretching vibration), strong absorption bands at $1711 \mathrm{~cm}^{-1}$ (C-O stretching vibration), $1267 \mathrm{~cm}^{-1}$ (C-O bonds symmetric stretching vibration of C-O bonds for alcohols), 1101 (bending vibration at the surface of adjacent hydrogen atoms on the phenyl ring bonds) and $727 \mathrm{~cm}^{-1}$ (bending vibration absorption of $\mathrm{CH}$-plane of the benzene ring bonds) [37]. In addition, weak band at $1450 \mathrm{~cm}^{-1}$ (vibrations of the angular deformation of $\mathrm{CH}_{2}$ ) was detected [34]. The absorption band at approximately $1016 \mathrm{~cm}^{-1}$ that is attributed to the extent of deformation and bending of $\mathrm{C}-\mathrm{O}-\mathrm{C}$ and $\mathrm{OH}$ groups of the starch structure $\left(999 \mathrm{~cm}^{-1}\right)$ was detected. In addition, a large band in the $3350 \mathrm{~cm}^{-1}$ region that is typically associated with TPS structure was not found [38]. The PE-BIO film IR spectrum without exposure to UV-B radiation presented the characteristic carbonyl group IR band $\left(1640 \mathrm{~cm}^{-1}\right)$ which indicated that the PE-BIO content is oxidized during the manufacturing process [26].

In particular, the ketone carbonyl and vinyliene indices are used to measure the degree of films degradation. The calculated $\mathrm{KC}$ bond indices from the FTIR spectra are found to be below 0.25 . TPS on the other hand exhibited a much higher $\mathrm{KC}$ bond index of 3.2. In literature, films containing mainly $\mathrm{PE}$ were found to exhibit $\mathrm{KC}$ bond index around 1 which means that PE containing blends are more degradable compared to polypropylene (PP) [39]. An increase in the KC bond indices was observed for LLDPE and PSW blends with TPS and PDQ-M (97 wt.\%/ 3wt.\%) compared to LLDPE (100) and PSW (100). This is caused by the PDQ-M and TPS in the LLDPE and PSW films which cleaved the polymer chain, enhancing chain movement and leading to an increase in crystallinity [40]. The smaller double bond indices seen for some blends in comparison to others may suggest that the double bond was used in polymer crosslinking [40]. The effect of additives in increasing $\mathrm{KC}$ bond index was also observed in the study of Portillo et al. [36] where Carbonyl Index for PE+AD (AD is oxo-degradable additive) was found to be greater than $\mathrm{PE}$. The KC bond index of unexposed LDPE was also reported in literature to be greater than HDPE and PE-OXO as it is a branched material with a greater number of tertiary carbon which makes it more prone to undergo photooxidation reactions [26]. The ketone carbonyl and vinylidene indices of LLDPE compounded in SSE were 0.11 and 0.12 , respectively. The ketone carbonyl and vinylidene indices of LLDPE compounded in TSE were 0.07 and 0.08 , respectively, and those of LLDPE compounded in BF/SSE were 0.06 and 0.075 , respectively. The indices of LLDPE compounded in TSE and BF/SSE were in agreement to the ketone carbonyl and vinylidene indices of PE (0.08 and 0.09, respectively) [40]. The promotion of this research work, namely considering hydro-biodegradable plastics is considered to be quite topical as of late after the restriction of the use of oxo-biodegradable [41]. Following the research conducted by our group [42], [43] and the continuation of the research efforts in this communication, legislations are expected to presented and formulated by local authorities to dictate the use of both oxo and hydro-biodegradable plastics. These will also eliminate possible threats of fragmentation and environmental pollution in eth near future [42]-[45].

\section{CONCLUSION}

Various plastic blends encompassing pro-degradants and thermoplastic starch resin, where compared with virgin polyethylene, plastic solid waste and thermoplastic starch films. Compounding was achieved using single, twin and blow film extrusion to produce $100 \mu \mathrm{m}$ thinness films which were tested for mechanical integrity and thermal properties. Thermogravimetry showed a single stage loss to all materials with the exception of the 
starch based compound indicting loss of polyester and starch. On the other hand, the samples showed minimal changes with respect to compounding technique and chemical fingerprinting using infrared spectroscopy. Furthermore, the materials were of acceptable market properties that allowed comparison of mechanical properties to typical plastic film products. This points towards the fact that biodegradable plastic waste blends could be utilised in the future as a standalone product to reduce dependency on virgin plastic materials.

\section{ACKNOWLEDGEMENTS}

The authors are grateful to the Kuwait Institute for Scientific Research (KISR) for supporting the activities of project EM065K. We acknowledge the financial support given by the Kuwait Foundation for the Advancement of Sciences (KFAS) towards the scientific mission to deliver the oral presentation of this communication.

\section{REFERENCES}

[1] Al-Salem, S., Al-Hazza'a, A., Karam, H., Al-Wadi, M., Al-Dhafeeri, A. \& AlRowaih, A., Insights into the evaluation of the abiotic and biotic degradation rate of commercial pro-oxidant filled polyethylene (PE) thin films. J. Environ. Manage., 250, 109475, 2019.

[2] Parker, L., Fast facts about plastic pollution, 2018. https://news.nationalgeographic.com/2018/05/plastics-facts-infographics-oceanpollution/.

[3] Boomerang Alliance, Marine plastic pollution by the numbers, 2016. https://www.boomerangalliance.org.au/sources.

[4] Colín-Chávez, C. et al., The release of carotenoids from a light-protected antioxidant active packaging designed to improve the stability of soybean oil. Food Bioprocess Technol, 7, pp. 3504-3515, 2014.

[5] Al-Salem, S.M., Establishing an integrated databank for plastic manufacturers and converters in Kuwait. Waste Manage., 29, pp. 479-484, 2009.

[6] Tilstra, L. \& Johnsonbaugh, D., The biodegradation of blends of polycaprolactone and polyethylene exposed to a defined consortium of fungi. J Environ Polym Degr, 1, pp. 257-267, 1993.

[7] Guarás, M.P., Alvarez, V.A. \& Ludueña, L.N., Processing and characterization of thermoplastic starch/polycaprolactone/compatibilizer ternary blends for packaging applications. J Polym Res, 22, p. 165, 2015.

[8] Alariqi, S.A.S. \& Singh, R.P., Effect of $\gamma$-Dose rate on biodegradation of $\gamma$-sterilized biomedical polyolefins. J Polym Environ, 18, pp. 600-607, 2010.

[9] Al-Salem, S.M. et al., Study of the degradation profile for virgin linear low-density polyethylene (LLDPE) and polyolefin (PO) plastic waste blends. J Mater Cycles Waste Manag, 21, pp. 1106-1122, 2019.

[10] Lee, J.A., Kontopoulou, M. \& Parent, J.S., Time and shear dependent rheology of maleated polyethylene and its nanocomposites. Polymer, 45, pp. 6595-6600, 2004.

[11] Wood-Adams, P.M., Dealy, J.M., DeGroot, A.W. \& Redwine, O.D., Effect of molecular structure on the linear viscoelastic behavior of polyethylene. Macromolecules, 33(20), pp. 7489-7499, 2000.

[12] Wood-Adams, P.M. \& Dealy, J.M., Using rheological data to determine the branching level in metallocene polyethylenes. Macromolecules, 33(20), pp. 74817488, 2000. 
[13] Dumoulin, M.M., Utracki, L.A. \& Carreau, P.J., Rheological properties of LLDPE/PP blends I. Dependence of tensile properties on composition. Progress and Trends in Rheology II, eds, H. Giesekus \& M.F. Hibberd, Steinkopff: Heidelberg, 1988.

[14] Naddeo, C., Guadagno, L., De Luca, S., Vittoria, V. \& Camino, G., Mechanical and transport properties of irradiated linear low density polyethylene (LLDPE). Polym Degrad Stab, 71, pp. 239-247, 2001.

[15] Biswas, J. et al., Linear low density polyethylene (LLDPE)/zeolite microporous composite film. Macromol. Res., 11, pp. 357-367, 2003.

[16] Pang, M.M., Pun, M.Y. \& Ishak, Z.A.M., Thermal, mechanical, and morphological characterization of biobased thermoplastic starch from agricultural waste/polypropylene blends. Polym Eng Sci, 54, pp. 1357-1365, 2014.

[17] Osman, H. \& Zakaria, M.H., Effects of durian seed flour on processing torque, tensile, thermal and biodegradation properties of polypropylene and high density polyethylene composites. Polym Plastic Tech Eng, 51, pp. 243-250, 2012.

[18] Azhari, C.H. \& Wong, S.F., Morphology-mechanical property relationship of polypropylene/starch blends. Pakistan J Bio Sci, 4, pp. 693-695, 2001.

[19] Berruezo, M., Ludueña, L.N., Rodriguez, E. \& Alvarez, V.A., Preparation and characterization of polystyrene/starch blends for packaging applications. J Plastic Film Sheet, 30, 2014.

[20] Elanmugilan, M., Sreekumar, P.A., Singha, N.K., Al-Harthi, M.A. \& De, S.K., Aging of low-density polyethylene in natural weather, underground soil aging and sea water: Effect of a starch-based prodegradant additive. Polym Eng Sci, 53, pp. 2389-2397, 2013.

[21] Beg, M.D.H. \& Pickering, K.L., Accelerated weathering of unbleached and bleached Kraft wood fibre reinforced polypropylene composites. Polym Degrad Stab, 93, pp. 1939-1946, 2008.

[22] Vogt, N.B. \& Kleppe, E.A., Oxo-biodegradable polyolefins show continued and increased thermal oxidative degradation after exposure to light. Polym Degrad Stab, 94, pp. 659-663, 2009.

[23] Al-Salem, S.M., Abraham, G., Al-Qabandi, O.A. \& Dashti, A.M., Investigating the effect of accelerated weathering on the mechanical and physical properties of high content plastic solid waste (PSW) blends with virgin linear low density polyethylene (LLDPE). Polym Test, 46, pp. 116-121, 2015.

[24] Al-Salem, S.M., Al-Dousari, N.M., Joseph Abraham, G., Aromin D'Souza, M., AlQabandi O.A. \& Al-Zakri W., Effect of die head temperature at compounding stage on the degradation of linear low density polyethylene/plastic film waste blends after accelerated weathering. Int J Polymer Sci, 5147209, 2016.

[25] La Mantia, F.P., Recycling of heterogeneous plastics wastes: I-Blends with lowdensity polyethylene. Polym Degrad Stab, 37, pp. 145-148, 1992.

[26] Martínez-Romo, A., González-Mota, R., Soto-Bernal, J.J. \& Rosales-Candelas, I., Investigating the degradability of HDPE, LDPE, PE-BIO, and PE-OXO films under UV-B radiation. $J$ Spec, 586514, 2015.

[27] George, S.C. \& Thomas, S., Transport phenomena through polymeric systems. Prog Polym Sci, 26, pp. 985-1017, 2001.

[28] Ojeda, T.F.M. et al., Abiotic and biotic degradation of oxo-biodegradable polyethylenes. Polym Degrad Stab, 94, 2009.

[29] Xingzhou, H., Wavelength sensitivity of photo-oxidation of polyethylene. Polym Degrad Stab, 55, pp. 131-134, 1997. 
[30] Gugumus, F., Contribution to the photolysis of hydroperoxides in polyethylene. Polym Degrad Stab, 27, pp. 19-34, 1990.

[31] D'Amelia, R.P., Gentile, S., Nirode, W.F. \& Huang L., Quantitative analysis of copolymers and blends of Polyvinyl Acetate (PVAc) using Fourier Transform Infrared Spectroscopy (FTIR) and Elemental Analysis (EA). World J Chem Edu, 4, pp. 25-31, 2016.

[32] Nazareth, M., Marques, M.R.C., Leite, M.C.A. \& Castro, Í.B., Commercial plastics claiming biodegradable status: Is this also accurate for marine environments? $J$ Hazard Mater, 366, pp. 714-722, 2019.

[33] Küpper, L., Gulmine, J.V., Janissek, P.R. \& Heise, H.M., Attenuated total reflection infrared spectroscopy for micro-domain analysis of polyethylene samples after accelerated ageing within weathering chambers. Vibrational Spec, 34, pp. 63-72, 2004.

[34] Nikolić, M.A.L et al., The challenges in lifetime prediction of oxodegradable polyolefin and biodegradable polymer films. Polym Degrad Stab, 145, pp. 102-119, 2017.

[35] Hayes, D.G. et al., Effect of diverse weathering conditions on the physicochemical properties of biodegradable plastic mulches. Polym Test, 62, pp. 454-467, 2017.

[36] Portillo, F., Yashchuk, O. \& Hermida, É., Evaluation of the rate of abiotic and biotic degradation of oxo-degradable polyethylene. Polym Test, 53, pp. 58-69, 2016.

[37] Finzi-Quintão, C.M., Novack, K.M. \& Bernardes-Silva, A.C., Identification of biodegradable and oxo-biodegradable plastic bags samples composition. Presented at Macro Symposia, 2016.

[38] Oliveira, T.A., Oliveira, R.R., Barbosa, R., Azevedo, J.B. \& Alves, T.S., Effect of reprocessing cycles on the degradation of PP/PBAT-thermoplastic starch blends. Carbohydrate Polym, 168, pp. 52-60, 2017.

[39] Moldovan, A., Paţachia Buican, R. \& Tierean, M.H., Characterization of polyolefins wastes by FTIR spectroscopy. Bullet Transilv, 5, 2012.

[40] Musioł, M., Rydz, J., Janeczek, H., Radeck, I., Jiang, G. \& Kowalczuk, M., Forensic engineering of advanced polymeric materials Part IV: Case study of oxobiodegradable polyethylene commercial bag - Aging in biotic and abiotic environment. Waste Mange, 64, pp. 20-27, 2017.

[41] European Commission (EC), report from the commission to the European parliament and the council on the impact of the use of oxo-degradable plastic, including oxodegradable plastic carrier bags, on the environment, 2018. http://ec.europa.eu/environment/circular-economy/pdf/oxo-plastics.pdf.

[42] Al-Salem, S.M., Influential parameters on natural weathering under harsh climatic conditions of mechanically recycled plastic film specimens. J Environ Mange, 230, pp. 355-365, 2019.

[43] Al-Salem, S.M. et al., Thermal response and degressive reaction study of oxobiodegradable plastic products exposed to various degradation media. Int. J. Polym. Sci., 9612813, 2019.

[44] Al-Salem, S.M., Uddin, S. \& Al-Yamani, F., An assessment of microplastics threat to the marine environment: A short review in context of the Arabian/Persian Gulf. Marine Environ Res, 159, 104961, 2020.

[45] Al-Salem, S.M., Uddin, S. \& Lyons, B., Evidence of microplastics (MP) in gut content of major consumed marine fish species in the State of Kuwait (of the Arabian/Persian Gulf). Marine Poll Bullt, 154, 111052, 2020. 